\title{
ON SOME PRODUCTS INVOLVING PRIMES
}

\section{S. UCHIYAMA}

ABSTRACT. Asymptotic formulae are given for the products $P_{l}(x)$ $(l=1,3)$ defined below.

We put, for $x \geqq 2$ and $l=1$ and 3 ,

$$
P_{l}(x)=\prod_{p \leq x ; p \equiv l(4)}\left(1-\frac{1}{p}\right),
$$

where the product is taken over the specified primes $p$. Our aim in the present note is to show that

$$
\begin{aligned}
& P_{1}(x)=\left(\pi A_{1} e^{-C}\right)^{1 / 2}(\log x)^{-1 / 2}+O\left((\log x)^{-3 / 2}\right), \\
& P_{3}(x)=\left(\frac{\pi A_{3} e^{-C}}{2}\right)^{1 / 2}(\log x)^{-1 / 2}+O\left((\log x)^{-3 / 2}\right),
\end{aligned}
$$

where $C$ denotes the Euler constant and

$$
A_{l}=\prod_{p \equiv l(4)}\left(1-\frac{1}{p^{2}}\right)
$$

(so that $A_{1} A_{3}=8 / \pi^{2}$ ).

Now, let us define $\chi(n)=0$ for even $n$ and $=(-1)^{(n-1) / 2}$ for odd $n$. Then, $\chi(n)$ is a residue character $(\bmod 4)$, and the corresponding $L$-series $L(s, \chi)=\sum_{n=1}^{\infty} \chi(n) n^{-8}$ represents a continuous function of $s$ for $s>0$. In particular, we have $L(1, \chi)=\pi / 4$ and

$$
L(1, \chi)=\prod_{p \leqq x}\left(1-\frac{\chi(p)}{p}\right)^{-1}+O\left(\frac{1}{\log x}\right)
$$

(cf. $[1, \S 109]$ ), whence

$$
\frac{P_{3}(x)}{P_{1}(x)}=\frac{\pi}{4} A_{3}+O\left(\frac{1}{\log x}\right) .
$$

On the other hand, we have by a well-known theorem due to F. Mertens (cf. $[1, \S 36]$ )

$$
P_{1}(x) P_{3}(x)=\frac{2 e^{-C}}{\log x}+O\left(\frac{1}{\log ^{2} x}\right) .
$$

Received by the editors October 22, 1970.

AMS 1970 subject classifications. Primary 10H25.

$K e y$ words and phrases. Dirichlet $L$-series. Mertens' theorem.

Copyright @ 1971, American Mathematical Society 
The result (1) follows at once from (2) and (3).

We note that our asymptotic formula for $P_{1}(x)$ will give a solution to a problem recently posed by D. Suryanarayana in [2].

\section{REFERENCES}

1. E. Landau, Handbuch der Lehre von der Verteilung der Primzahlen. Vols. 1, 2, 2nd ed., Chelsea Publishing Co., New York, 1953. MR 16, 904.

2. D. Suryanarayana, Problem 17 (1), Bull. Amer. Math. Soc. 76 (1970), 976.

SHINSHû University, MATSUMOTo, JAPAN 